\title{
Note taxonomique sur Camptocercus dadayi Stingelin, 1913, comb. nov. (Crustacea, Cladocera).
}

\author{
J. Rey 1 \\ E. Vasquez ${ }^{2}$ \\ Mots clés : Cladocera, taxonomie, Rio Uracoa, Vénézuéla.
}

Plusieurs specimens de Camptocercus du Rio Uracoa (nordest du Vénézuéla) ont été identifiés à Camptocercus australis var. dadayi décrit de Colombie par Stingelin (1913).

Sur la base de l'étude morphologique des individus vénézuéliens et des données de la littérature, le statut de ce taxon, d'abord rattaché à la forme australienne (australis), puis à la forme paléarctique (lilljeborgi) de Camptocercus, est discuté. L'ensemble des caractères propres aux populations sud-américaines permet de les séparer des formes australienne et paléarctique, et de leur attribuer un rang spécifique.

A taxonomic note on Camptocercus dadayi Stingelin, 1913, comb. nov. (Crustacea, Cladocera).

Keywords : Cladocera, taxonomy, Rio Uracoa, Venezuela.

Several specimens of Camptocercus from the Rio Uracoa (north-east Vénézuéla) have been identified as Camptocercus australis var. dadayi, previously described from Colombia by Stingelin (1913).

On the hasis of a morphological study of the Vénézuélan specimens and data from the literature, the status of this taxon is discussed, especially its relationship to the Australian form (austratis) and to the palaearctic form (lilljeborgi) of Camptocercts. The group of characters appropriate to the south american populations facilitates their separation from the australian and palaearctic forms and their assignment to a specific rank.

\section{Introduction}

L'examen de plusieurs Camptocercus australis récoltés dans le Rio Uracoa (nord-est du Vénézuéla) (Rey \& Vasquez 1986), identifiables à la variété dadayi decrite par Stingelin (1913) pour des specimens de Colombie, nous a amenés à revoir plus attentivement le statut de ce taxon.

L'étude morphologique des individus vénézuéliens, jointe aux données de la littérature, montre que les populations sud-américaines se caractérisent par une homogénéité de caractères qui les sépare net tement de la forme australienne (australis) et de la forme paléarctique (lillieborgi,, auxquelles ce taxon a été successivement rattaché.

\section{Description}

Camptocercus dadayi Stingelin, 1913 (fig. 1 à 13). = Camptocercus australis Daday, 1902 (pp. 266-269 ; . Tab. 10, fig. 2-5).

\footnotetext{
1. Laboratoire d'Hydrobiologie, UA CNRS 695, Université Paul Sabatier, 118, route de Narbonne, 31062 Toulouse Cé dex, France. 2. Fundación La Salle de Ciencias Naturales, Apdo 51, San Felix. Edo Bulivar, Vénézuẻla.
}

= Camptocercus australis Daday, 1905 (p. 189 ; Tab. 12, fig. 4)

? = Camptoce rcus australis var. Stingelin, 1905 (pp. 347, 348; Tab. 12, fig. 17)

= Camptocercus australis var. dadayi Stingelin, 1913 (pp. 620,621; fig. 21, 22).

= Camptocercus lilljeborgi var. dadayi Stingelin, 1913 (in Smirnov, 1971, p. 438, fig. 539).

MATERIEL

9 femelles parthénogénétiques, dont 5 femelles ovigères.

DATE ET STATION DE RECOLTE

13.07.1982.

Zone littorale du Rio Uracoa (catégorie des eaux noires)

Biotope à Eichhornia crassipes

pH : 5,7 ; température : $27,2^{\circ} \mathrm{C}$; conductivité : 33,4 $\mu \mathrm{s} \mathrm{cm}^{+1}$

\section{FEMELLES}

Longueur : $620 \mu$ à $750 \mu$.

Femelles ovigères : longueur minimale observée $620 \mu$ Valeurs moyennes mesurées $(n=8)$ :

Longueur: $665 \mu$.

Hauteur maximale : $408_{\mu}$. 
Hauteur minimale $(=$ bord postérieur libre des valves) : $171 \mu$.

Longueur/Hauteur maximale : 0,61 .

Longueur/Hauteur minimale : 0,26.

Contour général de la carapace : ovale allongé ( $f i g$. 1). Hauteur maximale des valves dans la moit ié antérieure du corps. Carène céphalique développée.

Angle postéro-ventral des valves très arrondi ( $f i g$. 2), dépourvu de denticules avec, sur la face interne, une rangée submarginale de courtes sétules atteignant la moitié de la hauteur postérieure des valves.

Apex du labre très largement arrondi, parfois légèrement crénelé avec, postérieurement, 1 ou 2 petites soies spiniformes (fig. 3-5). Antennule longue et mince (fig. 6), pourvue de 4 rangées de fines sétules.

Antennes bien développées. Soies antennai res distales 2 fois plus longues que les branches de l'antenne (fig. 7). Epine de l'article basal de l'exopode et épines terminales très courtes (fig. 8). Partie dis. tale du basipode et des articles antennaires finement denticulée.

Formule antennaire $: O(1), 0(0), 3(1) / O(0) .1(0) .3(1)$.

Postabdomen : 10 a 13 denticules marginaux allon. gés, triangulaires et aigus, de taille légèrement croissante distalement, serratés sur leur face concave ( $f \mathrm{ig}$ 9,10 ). 15 à 16 groupes latéraux de 4 à 5 (parfois 3 ) courtes soies triangulaires insérées un peu obliquement.

Griffe terminale longue (fig. 11), légèrement recourbę́e à son extrémité, avec, dans sa moitié proximale, une rangée de soies suivie de 3 denticules dont les 2 derniers sont beaucoup plus longs et plus largement séparés. Le denticule distal, le plus développé, est situé environ au milieu de la griffe. Epine basale assez courte et fine, portant proxima. lement une ciliature délicáte suivie de 3 sétules plus fortes et plus espacées bien visibles à l'immersion.

Branche externe de l'endite de la patte thoracique I à 3 soies : soie 1 courte, en crochet, soies 2 et 3 longues et épaisses, unisétulées, les 3 sétules proximales bien plus développées que les suivantes, qui diminuent progressivement de taille distalement (fig. 12, 13). Soie de l'exopodite nettement plus longue, garnie de sétules sur ses $2 / 3$ distals : partie basilaire relativement épaisse. La région distale de l'exopode porte 2 courtes soies triangulaires sur sa face anté. rieure (fig. 13).

\section{Discussion}

Par le contour général de la carapace, la forme du labre, l'armature du postabdomen, la structure de la griffe terminale, les individus du Rio Uracoa sont conformes à la variété dadayi créée par Stingelin(1913). Stingelin, en effet, ayant constaté une similitude de caractères (en particulier, structure de la griffe terminale, fine denticulation du bord postérieur des valves) chez les exemplaires colombiens et les Camptocercus australis Sars, figurés par Daday de Patagonie (Daday 1902) et du Paraguay (Daday 1905), considere que toutes ces formes représentent la variété sud-américaine de l'espèce australienne décrite par Sars (1896), et il les regroupe dans la variété dadayi.

Par la suite (Smirnov 1971), cette variété a été séparée à juste titre de Camptocercus australis qui diffère nettement par la griffe terminale à 1 seul denticule, le nombre de dents anales bien plus élevé et la forme allongée et triangulaire du labre (cf. Sars, 1896, PI.6, fig. 9 et 10 ; Smirnov et Timms, 1983, fig. $63 \mathrm{~B}, \mathrm{C} . \mathrm{E})$ et rattachée à l'espèce paléarctique lilljeborgi. L'attribution à cette dernière espèce apparaît plus discutable. Elle repose, en partie, sur un caractère ambigu : celui de la denticulation de l'angle postéro-ventral des valves, absente chez australis, mais représentée par des denticules distincts chez lilljeborgi.

Dans sa description de la variété dadayi, Stingelin parle bien de denticulation, encore qu'il précise " eine feine Randbezahnung ". Toutefois, la denticulation évoquée par cet auteur ne correspond pas à la denticulation observée chez lillieborgi. En fait, le même terme décrit 2 structures différentes.

Les spécimens colombiens ne présentent pas, en effet, une véritable denticulation, mais seulement une rangée postérieure de soies dont certaines sont plus développées et dentiformes, mais qui, toutes, sont nettement insérées submarginalement et sur la face interne de la région postéro-ventrale et postérieure des valves. La figure 21, p. 620, de Stingelin (1913) montre clairement cette structure.

L'espèce lilljeborgi, par contre, présente de véritables denticules, localisés à l'angle postéro-ventral de la carapace et prenant naissance à partir du bord même des valves. Une bonne figure en est donnée par Negrea (1983, p. 313, fig. 127 B). Cette dernière espèce se distingue encore par plusieurs autres caractères : 


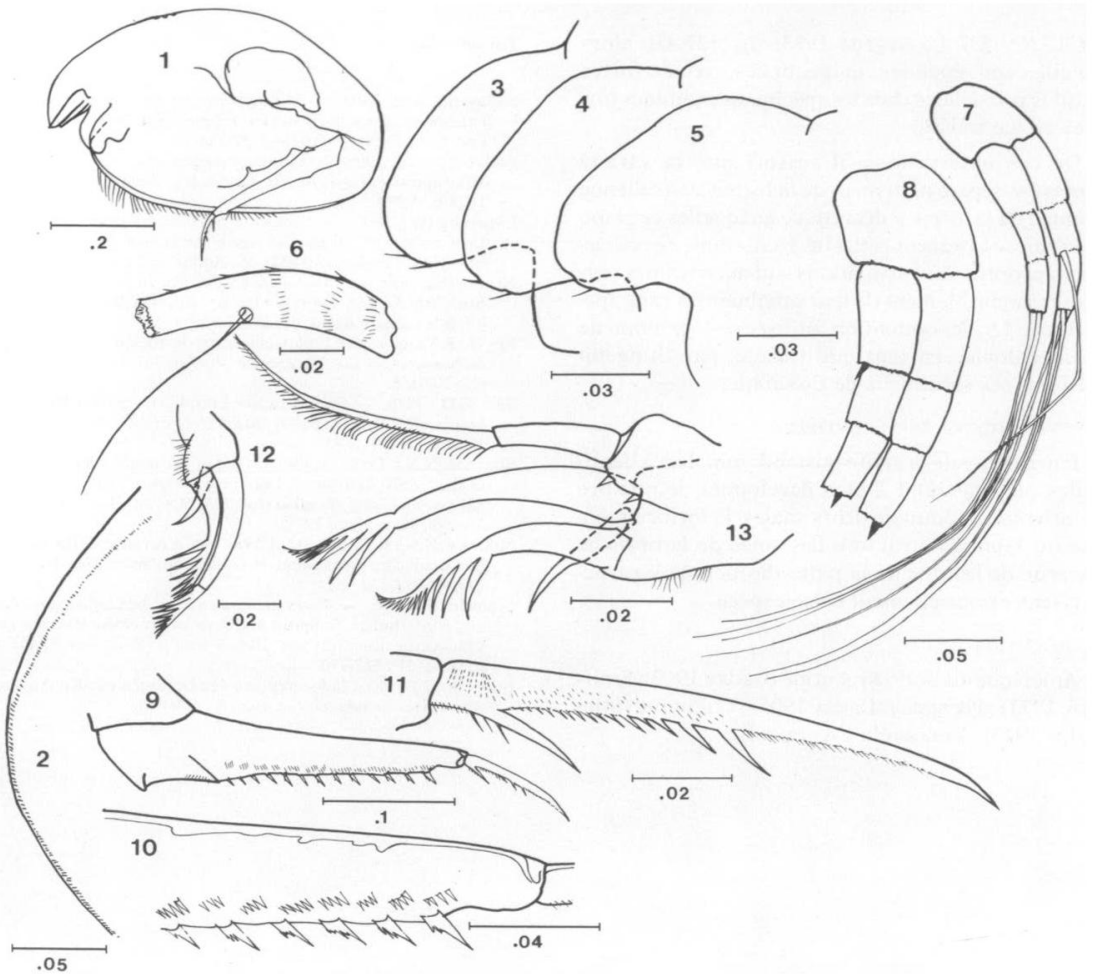

Fig. 1 a 13 : Camptocercus dadayi Stingelin, 19!3, comb. nov. Femelle parthénogénétique. 1 : Vue générale latérale. 2 : angle postéro-ventral, valve gauche, face inteme. 3 à $5:$ labre. $6:$ antennule (papilles sensorielles non figurées). 7 et $8:$ an tenne. 9: postabdomen. 10 : partie terminale du post-abdomen. 11 : griffe postabdominale. 12 : branche externe de l'endite de la patte thoracique 1. 13 : soies de la branche exteme de l'endite et exopodite de la patte thoracique 1.

- la griffe terminale munie d'un seul denticule médian :

- le nombre élevé de dents anales : 23 à 28 dents (cf. Lilljeborg 1901, fig. 4 et 10, Tab. 63 ; Flössner 1972, fig. 131 B ; Negrea 1983, fig. 127 I, H) contre 10 à 12 dents seulement chez les specimens de Patagonie (Daday 1902, Pl. 10, fig. 4), de Colombie (Stingelin 1913, p. 620, fig. 22) et du Vénézuéla (fig. 11, ce travail);

- le labre allongé, plus ou moins triangulaire, tout à fait distinct du labre plus court à apex largement arrondi que l'on observe chez les individus patagoniens (Daday 1902, Pl. 10, fig. 3), colombiens (Stingelin 1913. fig. 2!) et vénézuéliens (fig. 2 à 4, ce travail):

- la structure des soies de la branche externe de l'endite de la première patte thoracique: soie 1 nettement plus longue que chez dadayi (environ les $2 / 3$ de la plus longue soie), soies 2 et 3 longues, grêles, frangées de sétules courtes et fines (cf. Smirnov 
1971, fig. 537 I ; Negrea 1983, fig. 127 G), alors qu'elles sont épaisses, unipectinées avec de fortes sétules proximales chez les specimens examinés ( $f \mathrm{~g}$. 9 et 10 , ce travail).

De ces observations, il ressort que la variété dadayi se sépare nettement de la forme australienne comme de la forme paléarctique auxquelles ce taxon a été successivement rattaché. L'ensemble des caractères propres aux populations sud-americaines per. met raisonnablement de leur attribuer un rang spécifique. La dénomination utilisée est le nom de dadayi donné, en tant que variété, par Stingelin (1913) à ses spécimens de Colombie.

\section{BREve DIAGNOSE DIFFÉRENTIELLE}

L'armature de la griffe postabdominale à 3 dentjcules médians dont 2 bien développés, le nombre relativement réduit de dents anales, la forme arrondie du labre, la structure des soies de la branche externe de l'endite de la patte thoracique I, caractérisent essentiellement cette espèce.

\section{REPARTITION}

Amérique du Sud : Argentine (Daday 1902 ; Smir. nov 1971); Paraguay (Daday 1905); Colombie (St ingelin 1913); Vénézuéla (ce travail).

\section{Travaux cités}

Daday (E. von). 1902. - Mikroskopische Süsswassertiere aus Patagonien, gesammelt von Dr. Filippo Silvest ri im Jahre 1899 und 1900. Temész Füzeteck Budapest, 25:201-310.

Daday (E. von). 1905. - Untersuchungen über die Süsswasser Mikrotauna Paraguays. VII. Cladocera. Zoologica (Stuttgart). $18(44): 1-374$

Lilljeborg (W.). 1900. - Cladocera Sueciae. Nova Acta Soc. Sci. Upsala (Sêr. 3) : 701 pp. Fac simile Reissue of the original edi. tion with a Prologue (1982). W. Rodhe and D.G. Frey, eds.

Negrea (S.). 1983. - Crustacea. Cladocera. In Fanna Republicii Socialiste Románia, 4 (12) : 339 pp. Editura Academiei Republicii Socialiste Romania. Bucuresti.

Rey (J.) \& Vasquez (E.), 1986. Cladocères de quelques corps d'eaux du bassin moven de l'Orénoque (Vénézuéla). Annls Limnol., 22 (2) : $137-168$.

Sars (G.O.). 1896. - On freshwater Entomostraca from the neighbourhood of Sydney, partly raised from dried mud. Arch. Math. Natur, 18 (3) : $1-81$

Smirnov (N.N.), 1971, - Chydo ridae of the world's fauna. Fauna of the USSR. Crustacea, 1 (2), new series, $n^{\circ} 101$. Leningrad, 531 pp. (en russe). (English transl. A. Mercado. Israel Prog. Sci. Transl., Jerusalem, 1974).

Smirnov (N.N.) \& Timms (B.V.). 1983. - A revision of the Australian Cladocera (Crustacea). Records of the Australian Muse um. Suppl. 1: 1-132.

Stingelin (T.). 1905. - Untersuchungen über die Cladocerenfauna von Hinterindien, Sumatra und Java, nebst einem Beitrage zur Cladoceren Kenntnis der Hawaï Inseln. Zool. Jahrb. Abt. f. System, $21: 327.370$.

Stingelin (T.). 1913. Cladoceren aus den Gebirgen von Kolumbien. Mem. Soc. Neuchatel. Sci. nat., $5: 600-638$. 